\title{
Molecular targets and mechanism of action of dexmedetomidine in treatment of ischemia/reperfusion injury (Review)
}

\author{
YE CAI, HUI XU, JIA YAN, LEI ZHANG and YI LU \\ Ninth People's Hospital, Shanghai Jiao Tong University School of Medicine, Shanghai 200011, P.R. China
}

Received July 10, 2013; Accepted February 17, 2014

DOI: $10.3892 / \mathrm{mmr} .2014 .2034$

\begin{abstract}
Dexmedetomidine (DEX), a highly specific $\alpha_{2}$-adrenergic agonist, which exhibits anaesthetic-sparing, analgesia and sympatholytic properties. DEX modulates gene expression, channel activation, transmitter release, inflammatory processes and apoptotic and necrotic cell death. It has also been demonstrated to have protective effects in a variety of animal models of ischemia/reperfusion (I/R) injury, including the intestine, myocardial, renal, lung, cerebral and liver. The broad spectrum of biological activities associated with DEX continues to expand, and its diverse effects suggest that it may offer a novel therapeutic approach for the treatment of human diseases with $\mathrm{I} / \mathrm{R}$ involvement.
\end{abstract}

\section{Contents}

\section{Introduction}

2. Molecular mechanisms of ischemia/reperfusion (I/R) injury

3. Dexmedetomidine (DEX) mechanisms of action

4. Genetic alterations

5. Ion channels

6. Signaling pathways

7. Transmitter release

8. Inflammatory response

9. Role of DEX in I/R injury

10. Intestinal $\mathrm{I} / \mathrm{R}$

11. Myocardial I/R

12. Renal protection

13. Lung protection

14. Cerebral injury

15. Spinal injury

16. Other organ injuries

17. Conclusions

Correspondence to: Dr Hui Xu, Ninth People's Hospital, Shanghai Jiao Tong University School of Medicine, 639 Zhi Zao Ju Road, Shanghai 200011, P.R. China

E-mail:mzkxuhui@sina.cn

Key words: dexmedetomidine, ischemia/reperfusion, treatment

\section{Introduction}

Dexmedetomidine [DEX; (S)-4-[1-(2,3-dimethylphenyl) ethyl]-3H-imidazole], is a selective and potent $\alpha_{2}$-adrenergic receptor $\left(\alpha_{2}-\mathrm{AR}\right)$ agonist, which was approved by the US Food and Drug Administration in 1999 for sedation of patients hospitalized in intensive care units (ICUs). Since then, a growing number of research articles have emerged reporting other possible applications $(1,2)$, including use as a protective agent for ischemia/reperfusion ( $\mathrm{I} / \mathrm{R})$ injury in various organs. Initially, DEX represented a suitable sedative for the postoperative period and ICUs. A major goal of sedative treatment for patients in the postanesthesia care unit and the neurological ICU is to provide anxiolysis and analgesia (3). Thus, the use of $\alpha_{2}$-AR agonists is widely accepted in neuroanesthesia. It is also helpful to avoid additional cardiorespiratory workload and metabolic alterations caused by increased levels of catecholamines and other stress hormones. Additionally, DEX and other $\alpha_{2}$-AR agonists have been used to manage the sympathetic hyperactivity resulting from drug withdrawal syndromes in patients with substance abuse (4). Furthermore, $\alpha_{2}$-AR agonists have a potential application as prophylactic agents in neuroprotection following neuroanesthesia and neurointensive care, which has attracted much research interest in their role in $\mathrm{I} / \mathrm{R}$ injury in the brain and other critical organs.

$\alpha_{2}$-ARs have three subtypes (A, B and C) and are widely distributed in the nervous system. The $\alpha_{2 \mathrm{~A}}$-AR and $\alpha_{2 \mathrm{C}}$-AR subtypes appear to dominate in the central nervous system (CNS), while the $\alpha_{2 B}$-AR subtype is present at a low concentration in specific areas of the CNS and in the majority of peripheral tissues (5). DEX has been shown to exert protective effects on I/R injury in several organs/regions, including the intestines, heart, kidneys, lungs and liver. To the best of our knowledge, there have been no comprehensive reviews on the therapeutic value of DEX in I/R treatment.

Therefore, the aim of this review was to present the current state of knowledge on the effects of DEX on various I/R conditions and to highlight its potential application in $\mathrm{I} / \mathrm{R}$ injury treatment.

\section{Molecular mechanisms of $I / R$ injury}

In organ transplantation and other clinical settings, I/R injury is referred to as the process in which injury occurs by initial hypoxia and adenosine triphosphate (ATP) depletion at the 
time of organ procurement, with subsequent return of oxygen supply and blood flow at the time of reperfusion. The return of blood flow causes further organ damage through oxidative stress (OS) and the presence of pro-inflammatory chemokines and cytokines, a process that is referred to as the injury hypothesis' $(6,7)$. OS is defined as an overproduction of reactive oxygen species (ROS) that outnumber antioxidant defenses, which is implicated in pathobiological processes associated with the oxidation of biological macromolecules, including proteins, DNA and lipids. Increased OS is a well-known feature of renal I/R injury.

$\mathrm{I} / \mathrm{R}$ injury rapidly promotes the generation of superoxide and other ROS, including hydrogen peroxide and hydroxyl radicals. Studies on antioxidant treatments suggest that an early increase in ROS levels contributes to tissue damage and to the loss of function in cisplatin-, mercury-, glycerolor I/R-induced models of acute kidney injury (8). With a variety of forms of ischemic and toxic tissue injury, cellular accumulation of calcium ions $\left(\mathrm{Ca}^{2+}\right)$ and generation of oxygen free radicals have adverse effects on cellular and mitochondrial membranes. Damage to the mitochondria, resulting in impaired ATP synthesis and diminished activity of cellular energy-dependent processes, contributes to cell death (9). The accumulation of ROS, $\mathrm{Ca}^{2+}$ and leukocytes in ischemic tissues leads to end organ damage and ultimately to organ failure due to hemorrhagic shock (10).

\section{DEX mechanisms of action}

DEX regulates a number of physiological functions, including neurotransmitter release, insulin secretion, vasoconstriction, renal sodium reabsorption and intestinal chloride secretion, through the facilitation of chemical channels and ultimately by modulating genetic regulation.

\section{Genetic alterations}

At doses shown to be neuroprotective, DEX inhibits c-Fos and 70 kilodalton heat shock protein (hsp70) messenger RNA (mRNA) expression and enhances the nerve growth factor-induced gene A mRNA expression in the post-ischemic hippocampi of gerbils (11). Reduced gene expression of c-Fos and hsp70 were detected in CA1 pyramidal cells, which are prone to ischemic degeneration. An increased gene expression of nerve growth factor-induced gene A was observed in the CA3 and dentate gyrus, areas which are relatively resistant to ischemia. These alterations in early gene expression suggest that those mechanisms mediate the neuroprotective effects of $\alpha_{2}$-AR agonists (11).

Muszkat et al (12) reported 24 polymorphisms, including 16 novel variants, in the $\alpha_{2 \mathrm{~B}}$-AR and four common haplotypes with variable ethnic distributions. One haplotype containing the G-429T variant in the core promoter was associated with a significant reduction of DEX-induced venoconstriction. This haplotype was observed in $18 \%$ of African-American, but not in caucasian individuals.

However, Yağar et al (13) suggested that there is a weak effect of the $\alpha_{2 \mathrm{~A}}$-AR gene polymorphism in the response to DEX. The authors also reported the need for further clinical investigations to determine whether other polymorphisms of this gene or haplotype influence the sedation response and haemodynamic parameters in patients receiving DEX. The genotypes and haplotypes identified may provide useful information for future studies to examine physiological and pharmacological consequences of genetic variations in the $\alpha_{2}-\mathrm{AR}$, and the linkage to the physiological responses to DEX.

A study showed that the effect of DEX on reducing inflammation in sepsis may be involved in the epigenetic regulation of cytokine expression (14). The $\alpha_{2}-\mathrm{AR}$ is a $\mathrm{G}$ protein-coupled receptor that transduces signals by catalyzing the dissociation of the $G$ protein and $G$ protein subunits, thus modulating the subunits' downstream effectors. G proteins bind to the $\mathrm{C}$ terminus of histone deacetylase (HDAC) 5. DEX regulates the HDAC 2 and HDAC5 mRNA expression, and the histone $\mathrm{H} 3$ acetylation through $\alpha_{2}$-AR (14). Previous reports have shown that DEX-induced cell survival is associated with a decrease in cleaved caspase-3 levels and a reduction of high-mobility group protein B1 (HMGB1) release $(15,16)$. This confirms that DEX, at clinical doses, significantly inhibits HMGB1 translocation from the nucleus to the cytoplasm and lipopolysaccharide (LPS)-induced increases in HMGB1 mRNA expression in LPS-activated macrophages (17).

\section{Ion channels}

DEX binds to $\alpha_{2}$-ARs on the cell membrane of neurons of the locus coeruleus, leading to opening of inwardly rectifying potassium (IRK) channels, resulting in the hyperpolarization of the membrane (18). DEX inhibits paraventricular nucleus magnocellular neurons by activating the $G$ protein-coupled IRK current and inhibiting parvocellular neurons of the paraventricular nucleus by suppressing the hyperpolarization-activated current (19). Blockage of these currents may thus be a potential mechanism by which DEX depresses neuronal excitability (20).

\section{Signaling pathways}

DEX modulates a number of pathways involving $G$ protein-coupled receptors. $\alpha_{2 B}-A R$ stimulates protein kinase $\mathrm{C}(\mathrm{PKC})$ activity and inositol triphosphate production in renal distal convoluted tubule cells. This demonstrates that PKC is important for ischemic preconditioning since it activates the intracellular signaling pathways providing organ protection, the opening of sarcolemmal and mitochondrial ATP-sensitive $\mathrm{K}^{+}$channels and through gene transcription, promotes the protective cellular protein synthesis (21). DEX reverses the suppression of HSP27 phosphorylation by the activation of the adenylyl cyclase-cyclic adenosine monophosphate (cAMP) system in C6 cells, suggesting that DEX may have a neuroprotective effect through the modification of PKC activation-induced HSP27 phosphorylation (22). The $\alpha_{2}$-AR-focal adhesion kinase-Src-phosphatidylinositol 3-kinase (PI3K)-protein kinase B (Akt) $(23,24)$, and the imidazoline $I_{1}$ receptor-extracellular signal-regulated kinase 1/2 (ERK1/2)-mitochondrial ATP-sensitive $\mathrm{K}^{+}$channel pathways (25) are involved in the preconditioning and postconditioning effects of DEX against hippocampal oxygen and glucose deprivation-induced injury (26). Treatment with DEX reduces cerebral injury in rats exposed to transient focal 
$\mathrm{I} / \mathrm{R}$, which is mediated by the activation of the PI3K/Akt and ERK1/2 pathways, as well as the phosphorylation of downstream glycogen synthase kinase 3 (27). In addition, DEX attenuates mouse hippocampal CA1 long-term potentiation. $\alpha_{2}$-ARs and imidazoline $\mathrm{I}_{2}$ receptors are affected by DEX. The observation that imidazoline $\mathrm{I}_{2}$ receptors are involved in the DEX-mediated long-term potentiation reduction provides novel information on the basic actions of DEX in the CNS (28). Epidermal growth factor receptor transactivation in astrocytes in the mature brain in vivo represents an important process in response to $\alpha_{2}$-AR stimulation, and it may lead to ERK1/2 phosphorylation in astrocytes and adjacent neurons (29). The $\alpha_{2}$-ARs also activate the mitogen-activated protein kinase pathway and thus facilitate the proliferation of cells derived from human intestinal epithelium, as well as the proximal tubule of rats and pigs $(30,31)$.

A possible mechanism underlying the protective effects of DEX against pulmonary edema is the involvement of the L-arginine-nitric oxide pathway in $\alpha$-naphthylthiourea (ANTU)-induced acute lung injury (ALI). A nitric oxide synthase inhibitor, NG-nitro-L-arginine methyl ester, used prior to the administration of ANTU, significantly decreased the development of pleural effusion and the lung weight/body weight ratio (32). Thus, DEX may diminish acute $N$-methyl-D-aspartic acid-induced perturbation of neurotransmission (33). DEX induces the contraction of aortic rings through the activation of lipoxygenase and cyclo-oxygenase pathways, which is attenuated by an increased NO production (34).

DEX has a tandem role in pore domains in the weak IRK acid-sensitive $\mathrm{K}^{+}$channel 1 in the modulation of the function of the adrenergic nuclei locus coeruleus and/or other neuronal systems (35). Through $\alpha_{2 \mathrm{~A}}$-AR, DEX may activate astrocytes and promote the release of glial cell line-derived neurotrophic factors to protect neurons following stroke; this signaling is possibly dependent on the activation of the PKC and cAMP response element binding protein (36). Orexinergic neurons have been suggested to be involved in the regulation of the sleep-wake cycle. Supraclinical concentrations of DEX increase norepinephrine (or noradrenaline, NA) release from rat cerebrocortical slices, and this release may be mediated via orexin-1, but not $\alpha_{2}$-ARs. As $\alpha_{2}$-AR agonists also produce sedation and anesthesia, there may be a significant interaction between $\alpha_{2}$-AR agonists and the orexinergic system (37).

\section{Transmitter release}

DEX attenuates the ischemia-induced excessive NA release by activating presynaptic $\alpha_{2}$-ARs $(38,39)$, which may lead to the formation of free radicals (40). In addition, the protective action of $\alpha_{2}$-AR agonists may be due to a postsynaptic reduction in neuronal excitability and/or a possible presynaptic decrease in glutamate release (41-44), which is linked to the suppression of voltage-dependent $\mathrm{Ca}^{2+}$ channels and mitogen-activated protein kinase activity (42). However, DEX does not suppress the elevation in NA or glutamate levels in the brain associated with cerebral ischemia (45). Engelhard et al (45) show that the increase in circulating catecholamine concentrations during cerebral ischemia is suppressed by DEX, thus suggesting that the neuroprotective effects of DEX are associated with the inhibition of circulating NA, but not presynaptic NA in the brain.

DEX also is distributed to several neurons other than noradrenergic neurons. Therefore, NA and $\alpha_{2}$-adrenergic drugs influence the release of numerous other neurotransmitters, including 5-hydroxytryptamine (5-HT), dopamine and acetylcholine (46). The concomitant use of the peripherally acting $\alpha_{2}$-AR antagonist MK-467 in dogs treated with DEX prevented major changes in plasma glucose, insulin, non-esterified fatty acids and lactate (47). These results indicate that DEX inhibits insulin secretion through an $\alpha_{2}$-AR and pertussis toxin-sensitive trimetric $\mathrm{G}$ protein-binding protein pathway, which involves the activation of the $\mathrm{K}^{+}$channel and inhibition of the $\mathrm{Ca}^{2+}$ channel (48). DEX decreases evoked glutamate release from hippocampal rat brain slices during either depolarization or hypoxic stress, but does not alter hypoxic conditions mediated by alterations of $\mathrm{Ca}^{2+}$ levels (44).

DEX decreases malondialdehyde levels and increases total glutathione levels. Glutathione is involved in DNA synthesis and repair, cellular metabolic functions, inactivation of toxic substances and the prevention of damage caused by free radicals (49). In rats, the increased reliance of spinal $\alpha_{2}$-ARs on cholinergic stimulation to cause analgesia following nerve injury reflects, in part, a shift from direct inhibition to direct excitation of spinal cholinergic neurons, which, in turn, depends on brain-derived neurotrophic factors and on an interaction with the stimulatory G protein (50). DEX induces shedding of heparin-binding EGF from astrocytes, which, in turn, transactivates EGF receptors and stimulates astrocytic c-Fos and FosB expression. In addition, released heparin-binding EGF protects neurons from injury caused by $\mathrm{H}_{2} \mathrm{O}_{2}$. The transactivation of DEX in the brain has been confirmed in vivo. EGF transactivation by 5 -HT2B receptor stimulation is responsible for upregulation of cytosolic phospholipase A2 in astrocytes by fluoxetine, an antidepressant and inhibitor of the serotonin transporter, which is also a specific 5-HT2B agonist (51).

\section{Inflammatory response}

The innate immune system includes biochemical and cellular defenses, covering barriers, including the endothelium and epithelium, and biological macromolecules, such as Toll-like receptors (TLRs), the nucleotide-binding oligomerization domain proteins (associated with inflammatory disorders, including inflammatory bowel disease) and the pyrin/NALP-3 (also known as cryopyrin) family (associated with autoinflammatory syndromes) $(52,53)$. In addition, the innate immune system includes specific cell types, such as phagocytic (macrophages and neutrophils) and natural killer cells, the complement system and other molecules, including cytokines/chemokines that coordinate the intricate processes of the host defense. DEX prevents I/R injury through its cooperation with the innate immune system. Studies have demonstrated that DEX suppresses the TLR4-mediated inflammatory circuitry. TLR4 expression is triggered through endogenous ligands, including damage-associated molecular patterns and cytokines (15).

The inhibitory effect of DEX on the production of tumor necrosis factor- $\alpha$ (TNF- $\alpha$ ) and interleukin (IL)- 6 following endotoxin injection is noteworthy. Circulating endotoxin 
induces the release of cytokines, including TNF- $\alpha$ and IL-6, which produce hypotension and metabolic acidosis (54). Studies have demonstrated that sedation with DEX significantly decreases cytokine production, including IL- $1 \beta$, TNF- $\alpha$, and IL-6, in critically ill patients (55) and that DEX decreases TNF- $\alpha$, IL- 1 and IL- 6 levels to a greater extent than propofol (56). However, other studies have also demonstrated that over-activation of $\alpha_{2}$-AR may cause excessive inflammatory cytokine release via intracellular signaling. $\alpha_{2}$-AR antagonists may have therapeutic potential in the treatment of the ALI/acute respiratory distress syndrome, in which endotoxemia has a major role in organ failure (57). Yohimbine, an $\alpha_{2}$-ARs antagonist, downregulates the inflammatory over-response in the lung, and palliates the severity of lung damage by blocking $\alpha_{2}$-AR on inflammatory cells (24).

It has also been reported that DEX reduces the expression of the pro-apoptotic protein B-cell lymphoma-associated X, as well as increasing the anti-apoptotic B-cell lymphoma 2 (Bcl-2) protein, thereby attenuating apoptosis by inhibiting the intrinsic apoptotic cascade activation (32). The authors consider it likely that PI3K-Akt activation is one of the survival cascades activated by DEX to induce cytoprotection. The PI3K-Akt pathway promotes cell survival by phosphorylating the pro-apoptotic Bcl-2-associated death promoter, and upregulating anti-apoptotic Bcl-2 and B-cell lymphoma extra large protein expression, thus inhibiting the caspase-controlled intrinsic apoptotic pathway.

DEX induces apoptosis of neutrophils and inhibits superoxide production by neutrophils in a dose-dependent manner. Underlying mechanisms are associated with the caspase cascade and mitochondrial trifunctional protein loss (58). In a study investigating the effects of clamping across the femoral artery and vein in an epigastric island skin flap model, significant increases in myeloperoxidase (MPO) activity levels and in the flap necrosis area were observed as a result of I/R. In agreement with these results, an investigation conducted by the authors also demonstrated that, prior to and following the ischemic insult, DEX administration, at doses of 10 and $30 \mu \mathrm{g} / \mathrm{kg}$, reduced tissue MPO activity levels. This reduction was observed at the end of a 12-h and at the end of a seven-day reperfusion (59). However, DEX, at clinically relevant doses, did not significantly affect the expression of inflammatory molecules in activated macrophages. By contrast, above clinically relevant doses, DEX has been demonstrated to have minor, but significant biphasic effects (inhibiting, then enhancing) on the regulation of the expression of inflammatory molecules, possibly through $\alpha_{2}$-ARs. However, as the magnitude of the changes was relatively low, these effects may not be clinically significant (60).

DEX has a toxic effect on cerebral neurons when it is administered centrally into the cerebrospinal fluid via the intracisternal route (61). Although the exact mechanisms remain to be elucidated, this neurotoxic effect of DEX may be attributed to a direct vasoconstrictive effect mediated by cerebral vascular $\alpha_{2}$-ARs. To safely administer this highly liposoluble-selective $\alpha_{2}$-AR agonist via the intrathecal route as an adjuvant agent, further studies using advanced pathological investigations are required (61). DEX (1, 10 and $100 \mathrm{ng} / \mathrm{ml}$; corresponding to 1-, 10- and 100-fold clinical plasma concentrations) inhibits the expression of inflammatory molecules in the activated microglia (62). Results also suggest that DEX is a potent suppressor of LPS-induced inflammation in the activated microglia (62). These findings suggest that one of the mechanisms of the anti-inflammatory effects of DEX may be the modulation of cytokine production by macrophages and monocytes. The study revealed that DEX decreased cytokine production in sepsis. Critically ill patients with sepsis and septic shock suffer from a high degree of stress due to pain and anxiety, and from organ-specific responses to sepsis. An important objective in the management of these patients is to achieve an adequate level of sedation and analgesia. Previous findings indicate that DEX prevents inflammatory effects in septic patients during sedation (63).

DEX has a significant anti-ulcerative effect in rat gastric tissue at all test doses, thus indicating that there is an association between anti-ulcerative effects and $\alpha_{2}$-AR activation. In rats administered with DEX, all antioxidant parameters, with the exception of catalase, are increased. By contrast, with the exception of MPO, all oxidant parameters are decreased. Therefore, oxidant/antioxidant parameters are likely to be involved in the mechanism underlying the anti-ulcer effect of DEX (64). Selective reversal of inflammatory hyperalgesia by epidural administration of DEX may be achieved at all ages; relatively low doses are effective in early life, but the therapeutic window is narrow. These data are valuable for the use and dosing of epidural $\alpha_{2}$-AR agonists in neonates and infants (65).

\section{Role of DEX in I/R injury}

In the majority of brain regions, epinephrine and NA appear to act as full agonists, whereas DEX is a partial agonist (66). However, DEX is a full agonist at the $\alpha_{2 \mathrm{~B}}$-AR and a partial agonist at the $\alpha_{2 \mathrm{~A}}-\mathrm{AR}$ and the $\alpha_{2 \mathrm{C}}-\mathrm{AR}$ (67). Regional differences in agonist-associated activity may be due to differences in $\alpha_{2}$-AR subtype distribution and/or regional differences in expression levels and subtypes of $\mathrm{Gi} / \mathrm{o}$ proteins to which $\alpha_{2}$-AR are coupled (66).

The decrease in blood flow varies greatly among specific organs due to DEX. While flow through arteriovenous anastomoses and skin is decreased by $70-90 \%$, renal blood flow is decreased by $30 \%$ and cerebral blood flow varied only when baseline blood flow was high, in this situation, the largest decrease in hemodynamic variables occurred. These data indicate that DEX causes considerable redistribution of blood flow, predominantly reducing blood flow to less vital organs and shunt flow (68). With this redistribution, DEX provides complete protection for various organs, aspects of which are presented in the following sections.

\section{Intestinal I/R}

Intestinal I/R often occurs in the clinic, including severe burns, trauma, septic shock and a number of other surgical procedures. Splanchnic circulation, the last to be restored following resuscitation, is particularly vulnerable with hypotension, which is primarily observed in surgical and anesthesiological complications. With its effect on the central $\alpha_{2}$-AR, DEX may attenuate the surgical stress and the microcirculatory blood flow intensity in intestinal mucosa and muscles (69). 
Previous studies have shown that $\alpha_{2}$-AR agonist-induced sedation, analgesia, hypotension and hypothermia are mediated by the $\alpha_{2 \mathrm{~A}}$-AR, while the $\alpha_{2 \mathrm{C}}$-AR mediates the $\alpha_{2}$-AR agonist effects on the startle reflex, the stress response and locomotion (70). Furthermore, surgical stress and pain are a stimulating factors, which activate the sympathetic nervous system and microthrombosis formation in several small vessels in the intestinal muscle, which, in turn, results in a conspicuous reduction of perfused small vessel density. DEX may reduce sympathetic nervous activity and result in vasodilation of small vessels. The restoration of intestinal microcirculation, including the normalization of global hemodynamics (69), helps to reduce the vascular bed in intestinal I/R.

In a recent study, it was reported that the dose-dependent administration of DEX prior to ischemia, but not following it, attenuates intestinal I/R-induced intestinal injury (32). DEX also attenuates intestinal mucosal epithelial cell apoptosis, as observed by decreases in the apoptotic index, caspase- 3 protein expression, intestinal injury and consequently rat mortality (32).

The authors conclude that DEX, acting at the $\alpha_{2 \mathrm{~A}}-\mathrm{AR}$, is likely to affect the encoding process to decrease discrete cue fear memory. However, its ability to suppress the contextual memory is likely to be the result of blocking the consolidation process. The ability of $\alpha_{2}$-AR agonists to suppress the fear memory may be a valuable clinical property to suppress memory formation during stressful situations (71).

\section{Myocardial I/R}

DEX, with its wide effect on inflammatory responses, has certain protective properties in myocardial I/R. However the timing of administration may have an important role in its protective mechanism. DEX administration prior to ischemia significantly reduced the infarct size in an isolated rat heart model of myocardial I/R injury (32), whereas DEX administration at the initiation of reperfusion with the same dose and in the same experimental model increased the myocardial infarct size (72). A stable plasma concentration of $0.5 \mathrm{ng} / \mathrm{ml}$ DEX in dogs with an artificial coronary stenosis decreased the myocardial ischemic load during the first $2 \mathrm{~h}$ of emergence from halothane anesthesia (73).

A more likely explanation for the cardioprotective effect of DEX is that the drug alters the myocardial oxygen balance $(74,75)$. The decrease in emergence-associated myocardial ischemic load caused by DEX may be explained by its sympatholytic effects, which improve the myocardial oxygen supply/demand ratio. DEX decreases the heart rate, which is is of pivotal importance in decreasing myocardial ischemia, since it improves the supply from a prolonged diastolic perfusion time and is the most important determinant of the myocardial oxygen demand. Thus, the decrease in myocardial ischemic load by DEX most likely results from its sympatholytic effects (73). With the exception of their central mediation of decreases in catecholamine, $\alpha_{2}$-AR agonists may also cause peripheral and coronary vasoconstriction by stimulating postjunctional $\alpha_{2}$-AR. Roekaerts et al (75) have provided evidence that $\alpha_{2}$-AR stimulation may beneficially modulate coronary blood flow during myocardial ischemia by preventing the transmural redistribution of blood flow away from the ischemic endocardium.

\section{Renal protection}

DEX treatment reduced mortality in septic mice (14). It also ameliorated sepsis-induced acute kidney injury by decreasing inflammatory cytokine expression, including plasma HMGB1 (15) and increasing the expression of bone morphogenetic protein 7 (14). Pretreatment with DEX also decreased TLR4 expression in tubular cells (15). Treatment with DEX resulted in normal glomeruli and slight edema of tubular cells (76). Pre- or post-treatment with DEX provided cytoprotection and improved tubular architecture and function following renal ischemia. Treatment of rats with DEX following acute hemorrhage resulted in improved renal function, but in higher tubular dilation scores (77). In anesthetized dogs, low doses of DEX inhibited vasopressin secretion, causing aqueous diuresis (78). By exerting these effects, DEX may protect kidneys during ischemic events.

Studies on the use of DEX during surgery of patients are limited. Patients who received DEX as part of their analgesic regimen had a significantly greater urine output for the first $24 \mathrm{~h}$ following surgery. More importantly, serum creatinine was significantly decreased in the DEX group, and this decrease was sustained for up to a week following surgery (79). In percutaneous nephrolithotomy, an intra-operative infusion of DEX was not observed to have beneficial effects on creatinine clearance, neutrophil gelatinase-associated lipocalin, or cystatin C levels following the procedure; however, renin levels were reduced (80). However, renin levels are susceptible to numerous factors in humans and, therefore, are not a gold standard treatment option. The traditional administration of DEX is associated with decreased renal vasoconstriction, which does not occur with rapid loading of DEX or an infusion of markedly high doses. DEX administration directly inhibits the release of renin in the kidney, which promotes renal arterial vasodilatation (80). DEX also reduces renal vascular resistance and increases the glomerular filtration rate and filtration fraction, thus promoting histological changes consistent with pathological features of ischemia (77). These findings support the hypothesis that DEX protects against renal injury caused by I/R.

\section{Lung protection}

DEX treatment has demonstrated a potential protective benefit on lungs by preventing ANTU-induced ALI in an experimental rat model and in intensive care patients (32), and by reducing post-pneumoperitoneal I/R injury in a ventilated rat model (69). DEX-ketamine combinations mitigate pulmonary inflammatory response-induced lung injury in endotoxemic rats, and decrease lung permeability and ALI development in rats with either hemorrhagic shock or intracranial hypertension (81). DEX may provide lung protection following renal $\mathrm{I} / \mathrm{R}$. Therefore, it may be concluded that DEX reduces renal and lung injury simultaneously following kidney I/R, in addition to its sedative and analgesic properties (55). However, a number of studies have demonstrated no change in lung histological results following intraperitoneal DEX administration (32), which may be associated with the timing and dosage of the administration.

A previous study investigated the efficacy of DEX in I/R injury prevention. Hanci et al (82) intraperitoneally 
administered DEX 30 min following the establishment of a testis torsion in rats. Biochemical and histopathological results showed that that DEX reduced I/R injury following $4 \mathrm{~h}$ reperfusion. Kocoglu et al (76) used intraperitoneal DEX (100 mg/kg) to prevent I/R-associated kidney injury during reperfusion 60 min following renal ischemia. As a main result, kidney tissue examined histopathologically following $45 \mathrm{~min}$ reperfusion exhibited decreased injury. Gu et al (83) found that significant injury developed in lungs of rats following renal $\mathrm{I} / \mathrm{R}$, and that DEX (25 mg/kg) administered intraperitoneally prior to and following ischemia, effectively prevented the damage (69).

\section{Cerebral injury}

DEX attenuated isoflurane-induced injury in the developing brain, providing neurocognitive protection (84). DEX may reduce anaesthetic isoflurane-induced neuroapoptosis in vivo and ganotypic hippocampal cultures in vitro (16). DEX exerted a protective effect on traumatically injured hippocampal cells with a maximum effect at a $1 \mu \mathrm{M}$ dose, which was partially reversed by the simultaneous administration of the ERK inhibitor PD98059. DEX exerted direct neuroprotective and antiapoptotic effects on cultured cortical neuronal cells when temperature, oxygen and glucose supply were closely controlled (16). In vivo, $25 \mathrm{mg} / \mathrm{kg}$ DEX inhibited isoflurane-induced cortical injury, but is was not possible to demonstrate whether higher doses provided a superior protection against isoflurane-induced apoptosis. Thus, DEX may reduce isoflurane-induced neuroapoptosis in the developing rat cortex and exert anti-apoptotic effects in vitro (16).

An increasing number of in vitro and in vivo studies indicates that DEX also exerts a cell-protective effect on nervous tissue under ischemic conditions $(16,35,85-88)$. The relative safety of DEX administered as a sedative agent to neonatal animals was associated with the development of hippocampal synaptic functions. DEX exhibited a preconditioning effect against ischemic injury in hippocampal slices subjected to oxygen and glucose deprivation (16). DEX prevented delayed neuronal death in the CA3 area and the dentate hilus in the gerbil hippocampus when administered prior to and following the induction of ischemia. Low doses of DEX (3 $\mu \mathrm{g} / \mathrm{kg}$ ) provided potent neuroprotective effects against cerebral ischemia (86). Co-administration of lidocaine and DEX improved the neurological outcome without the alteration of glutamate and NA levels during forebrain ischemia in rats (87). The DEX-hypothermia combination improved the short-term neurological outcome following unilateral transient forebrain ischemia in rats (88). DEX administration during mesenteric arterial occlusion decreased I/R-associated cellular damage (35). A preference for DEX as an anesthetic during the reperfusion procedure or following sedation during mechanical ventilation in the critical care unit has the potential to attenuate reperfusion injury. However, the clinical significance of these preliminary findings requires further investigation in human subjects (35).

Similar neuroprotective effects against excitotoxicity were also found in vitro in primary cortical neurocultures exposed to a neurotoxic concentration of $N$-methyl-D-aspartic acid. The neuroprotective effect was observed in the cortex and in the white matter, where cystic lesion formation was prevented.
Cystic white matter lesions account for an increasing number of permanent neurological disabilities in children born prematurely (89). These data suggest that DEX administration either prior to or immediately following brain injury has no effect on brain water content or short-term neurological outcomes in surgical brain injury. A previous study showed significant neurological improvement in DEX-treated animals following stroke (88). However, one particular study showed no difference in neurological outcome (90), which may be due to the larger area of injury, which created a marked deficit in surgical brain injury compared with other models. In addition, the treatment effect may have been too small for detection in this study.

\section{Spinal injury}

DEX infusion during multilevel spinal fusions moderately improves the quality of recovery and possibly reduces fatigue in the early postoperative period. DEX moderately enhances early recovery of patients following surgery, as measured by the Quality of Recovery-40 questionnaire and the medication moderately reduced fatigue as measured by the Fatigue Severity Scale (85). Thus, DEX may have a neuroprotective effect in spinal cord injury (SCI), which may be partially attributed to the lipid peroxidation inhibition (91), and which decreases inflammatory cytokines (92). Traumatic SCI leads to an increase in lipid peroxidation and decreases enzymatic or non-enzymatic endogenous antioxidative defense systems. SCI also leads to apoptosis in the spinal cord. DEX treatment prevents lipid peroxidation and augments endogenous antioxidative defense systems in cerebrospinal fluid or spinal cord tissue; however, it failed to prevent apoptosis or neurodeficits following traumatic SCI. Thus, DEX did not produce beneficial results in SCI in rabbits; however, further detailed experimental studies are required to clarify the medicinal effects in SCI (93). Treatment of mice with DEX preserved motoric function and neuronal viability following aortic cross-clamping. In addition, mice exhibited an almost complete reversal of the protective effect with the administration of the $\alpha_{2}$-AR antagonist atipamezole. DEX appears to attenuate spinal cord I/R injury via $\alpha_{2}$-AR-mediated agonism (94). In vivo studies showed that intrathecal DEX has no significant pathological impacts on the spinal cord, and in vitro experiments indicated that DEX exhibits potential protective effects on lidocaine-induced neuronal cell death (61).

\section{Other organ injuries}

DEX treatment prevented increases in serum superoxide dismutase and malondialdehyde activity levels, and decreased the erythrocyte deformability index when administered prior to portal clampage in liver I/R (89). DEX attenuated the liver damage associated with sepsis, shock and other diseases associated with local or systemic inflammation (54). In a clinical study, Venn et al (55) showed that DEX prevented I/R injury associated with a tourniquet in upper extremity surgery.

\section{Conclusions}

Recently identified biological roles of DEX demonstrate its multiple physiological functions. It exerts its effects via the 
mediation of transmitters, several signaling pathways and inflammatory responses. An increasing number of studies demonstrate the beneficial effects of DEX on I/R injury. Further studies are required to evaluate the effects of DEX in clinical settings and determine whether DEX is beneficial in various diseases and clinical conditions associated with I/R. This review shows that DEX may be novel avenue to I/R injury therapies in humans.

\section{References}

1. McCutcheon CA, Orme RM, Scott DA, Davies MJ and McGlade DP: A comparison of dexmedetomidine versus conventional therapy for sedation and hemodynamic control during carotid endarterectomy performed under regional anesthesia. Anesth Analg 102: 668-675, 2006

2. Ramsay MA and Luterman DL: Dexmedetomidine as a total intravenous anesthetic agent. Anesthesiology 101: 787-790, 2004

3. Billings FT 4th, Chen SW, Kim M, et al: alpha2-Adrenergic agonists protect against radiocontrast-induced nephropathy in mice. Am J Physiol Renal Physiol 295: F741-F748, 2008.

4. Multz AS: Prolonged dexmedetomidine infusion as an adjunct in treating sedation-induced withdrawal. Anesth Analg 96: 1054-1055, 2003.

5. Paris A, Mantz J, Tonner PH, Hein L, Brede M and Gressens P: The effects of dexmedetomidine on perinatal excitotoxic brain injury are mediated by the alpha2A-adrenoceptor subtype. Anesth Analg 102: 456-461, 2006.

6. Land W, Schneeberger H, Schleibner S, et al: The beneficial effect of human recombinant superoxide dismutase on acute and chronic rejection events in recipients of cadaveric renal transplants. Transplantation 57: 211-217, 1994.

7. Land WG: The role of postischemic reperfusion injury and other nonantigen-dependent inflammatory pathways in transplantation. Transplantation 79: 505-514, 2005.

8. O'Neill S, Ross JA, Wigmore SJ and Harrison EM: The role of heat shock protein 90 in modulating ischemia-reperfusion injury in the kidney. Expert Opin Investig Drugs 21: 1535-1548, 2012.

9. Malis CD and Bonventre JV: Mechanism of calcium potentiation of oxygen free radical injury to renal mitochondria. A model for post-ischemic and toxic mitochondrial damage. J Biol Chem 261: 14201-14208, 1986.

10. Wang QM, Stalker TJ, Gong Y, Rikitake Y, Scalia R and Liao JK: Inhibition of Rho-kinase attenuates endothelial-leukocyte interaction during ischemia-reperfusion injury. Vasc Med 17: 379-385, 2012

11. Wittner M, Sivenius J and Koistinaho J: Alpha2-adrenoreceptor agonist, dexmedetomidine, alters acute gene expression after global ischemia in gerbils. Neurosci Lett 232: 75-78, 1997.

12. Muszkat M, Kurnik D, Solus J, et al: Variation in the alpha2B-adrenergic receptor gene (ADRA2B) and its relationship to vascular response in vivo. Pharmacogenet Genomics 15: 407-414, 2005

13. Yağar S, Yavas $S$ and Karahalil B: The role of the ADRA2A C1291G genetic polymorphism in response to dexmedetomidine on patients undergoing coronary artery surgery. Mol Biol Rep 38: 3383-3389, 2011.

14. Hsing CH, Lin CF, So E, et al: $\alpha_{2}$-Adrenoceptor agonist dexmedetomidine protects septic acute kidney injury through increasing BMP-7 and inhibiting HDAC2 and HDAC5. Am J Physiol Renal Physiol 303: F1443-F1453, 2012.

15. Gu J, Sun P, Zhao H, et al: Dexmedetomidine provides renoprotection against ischemia-reperfusion injury in mice. Crit Care 15: R153, 2011.

16. Dahmani S, Rouelle D, Gressens P and Mantz J: Effects of dexmedetomidine on hippocampal focal adhesion kinase tyrosine phosphorylation in physiologic and ischemic conditions. Anesthesiology 103: 969-977, 2005.

17. Chang Y, Huang X, Liu Z, et al: Dexmedetomidine inhibits the secretion of high mobility group box 1 from lipopolysaccharide-activated macrophages in vitro. J Surg Res 181: 308-314, 2013.

18. Chiu TH, Chen MJ, Yang YR, Yang JJ and Tang FI: Action of dexmedetomidine on rat locus coeruleus neurones: intracellular recording in vitro. Eur J Pharmacol 285: 261-268, 1995.
19. Shirasaka T, Kannan $H$ and Takasaki M: Activation of a $\mathrm{G}$ protein-coupled inwardly rectifying $\mathrm{K}+$ current and suppression of Ih contribute to dexmedetomidine-induced inhibition of rat hypothalamic paraventricular nucleus neurons. Anesthesiology 107: 605-615, 2007.

20. Chen BS, Peng $\mathrm{H}$ and Wu SN: Dexmedetomidine, an alpha2-adrenergic agonist, inhibits neuronal delayed-rectifier potassium current and sodium current. Br J Anaesth 103: 244-254, 2009.

21. Curtis FG, Vianna PT, Viero RM, et al: Dexmedetomidine and $\mathrm{S}^{+}$-ketamine in ischemia and reperfusion injury in the rat kidney. Acta Cir Bras 26: 202-206, 2011

22. Tanabe K, Takai S, Matsushima-Nishiwaki R, Kato K, Dohi S and Kozawa O: Alpha2 adrenoreceptor agonist regulates protein kinase $\mathrm{C}$-induced heat shock protein 27 phosphorylation in C6 glioma cells. J Neurochem 106: 519-528, 2008.

23. Girault JA, Costa A, Derkinderen P, Studler JM and Toutant M: FAK and PYK2/CAKbeta in the nervous system: a link between neuronal activity, plasticity and survival? Trends Neurosci 22: 257-263, 1999.

24. Parcellier A, Tintignac LA, Zhuravleva E and Hemmings BA: PKB and the mitochondria: AKTing on apoptosis. Cell Signal 20: 21-30, 2008.

25. Dahmani S, Paris A, Jannier V, et al: Dexmedetomidine increases hippocampal phosphorylated extracellular signal-regulated protein kinase 1 and 2 content by an alpha 2-adrenoceptor-independent mechanism: evidence for the involvement of imidazoline I1 receptors. Anesthesiology 108: 457-466, 2008.

26. Dahmani S, Rouelle D, Gressens P and Mantz J: Characterization of the postconditioning effect of dexmedetomidine in mouse organotypic hippocampal slice cultures exposed to oxygen and glucose deprivation. Anesthesiology 112: 373-383, 2010.

27. Zhu YM, Wang CC, Chen L, et al: Both PI3K/Akt and ERK1/2 pathways participate in the protection by dexmedetomidine against transient focal cerebral ischemia/reperfusion injury in rats. Brain Res 1494: 1-8, 2013

28. Takamatsu I, Iwase A, Ozaki M, Kazama T, Wada K and Sekiguchi M: Dexmedetomidine reduces long-term potentiation in mouse hippocampus. Anesthesiology 108: 94-102, 2008.

29. Du T, Li B, Liu S, et al: ERK phosphorylation in intact, adult brain by alpha(2)-adrenergic transactivation of EGF receptors. Neurochem Int 55: 593-600, 2009.

30. Schaak S, Cussac D, Cayla C, et al: Alpha(2) adrenoceptors regulate proliferation of human intestinal epithelial cells. Gut 47: 242-250, 2000.

31. Cussac D, Schaak S, Gales C, Flordellis C, Denis C and Paris H: alpha(2B)-Adrenergic receptors activate MAPK and modulate proliferation of primary cultured proximal tubule cells. Am J Physiol Renal Physiol 282: F943-F952, 2002.

32. Hanci V, Yurdakan G, Yurtlu S, Turan IÖ and Sipahi EY: Protective effect of dexmedetomidine in a rat model of $\alpha$-naphthylthiourea-induced acute lung injury. J Surg Res 178: 424-430, 2012.

33. Niittykoski M, Haapalinna A and Sirviö J: Diminution of $\mathrm{N}$-methyl-D-aspartate-induced perturbation of neurotransmission by dexmedetomidine in the CA1 field of rat hippocampus in vitro. Neurosci Lett 281: 95-98, 2000.

34. Kim HJ, Sohn JT, Jeong YS, et al: Direct effect of dexmedetomidine on rat isolated aorta involves endothelial nitric oxide synthesis and activation of the lipoxygenase pathway. Clin Exp Pharmacol Physiol 36: 406-412, 2009.

35. Kiliç K, Hanci V, Selek S, et al: The effects of dexmedetomidine on mesenteric arterial occlusion-associated gut ischemia and reperfusion-induced gut and kidney injury in rabbits. J Surg Res 178: 223-232, 2012.

36. Yan M, Dai H, Ding T, et al: Effects of dexmedetomidine on the release of glial cell line-derived neurotrophic factor from rat astrocyte cells. Neurochem Int 58: 549-557, 2011.

37. Hirota K, Nomura H, Kudo M, Mori N, Kudo T and Kushikata T: Supraclinical concentrations of dexmedetomidine evoke norepinephrine release from rat cerebrocortical slices possible involvement of the orexin-1 receptor. Neurosci Lett 372: 142-145, 2004.

38. Globus MY, Busto R, Dietrich WD, Martinez E, Valdés I and Ginsberg MD: Direct evidence for acute and massive norepinephrine release in the hippocampus during transient ischemia. J Cereb Blood Flow Metab 9: 892-896, 1989. 
39. Matsumoto M,Zornow MH, Rabin BC and Maze M: The alpha 2 adrenergic agonist, dexmedetomidine, selectively attenuates ischemia-induced increases in striatal norepinephrine concentrations. Brain Res 627: 325-329, 1993.

40. Maier C, Steinberg GK, Sun GH, Zhi GT and Maze M: Neuroprotection by the alpha 2-adrenoreceptor agonist dexmedetomidine in a focal model of cerebral ischemia. Anesthesiology 79: 306-312, 1993.

41. Eser O, Fidan H, Sahin O, et al: The influence of dexmedetomidine on ischemic rat hippocampus. Brain Res 1218: 250-256, 2008.

42. Chiu KM, Lin TY, Lu CW and Wang SJ: Inhibitory effect of glutamate release from rat cerebrocortical nerve terminals by $\alpha 2$ adrenoceptor agonist dexmedetomidine. Eur J Pharmacol 670: 137-147, 2011.

43. Bickler PE and Hansen BM: Alpha 2-adrenergic agonists reduce glutamate release and glutamate receptor-mediated calcium changes in hippocampal slices during hypoxia. Neuropharmacology 35: 679-687, 1996.

44. Talke P and Bickler PE: Effects of dexmedetomidine on hypoxia-evoked glutamate release and glutamate receptor activity in hippocampal slices. Anesthesiology 85: 551-557, 1996.

45. Engelhard K, WernerC,Kaspar S, et al: Effect of the alpha2-agonist dexmedetomidine on cerebral neurotransmitter concentrations during cerebral ischemia in rats. Anesthesiology 96: 450-457, 2002.

46. Valtonen P, Haapalinna A, Riekkinen P Sr and Halonen T: Effect of alpha 2-adrenergic drugs dexmedetomidine and atipamezole on extracellular amino acid levels in vivo. Eur J Pharmacol 285: 239-246, 1995.

47. Kose EA, Bakar B, Kasimcan O, et al: Effects of intracisternal dexmedetomidine on cerebral neuronal cells in rat: a preliminary study. Turk Neurosurg 23: 38-44, 2013.

48. Kodera SY, Yoshida M, Dezaki K, et al: Inhibition of insulin secretion from rat pancreatic islets by dexmedetomidine and medetomidine, two sedatives frequently used in clinical settings. Endocr J 60: 337-346, 2013.

49. Kurt A, Ingec M, Isaoglu U, et al: An investigation about the inhibition of acute ischemia/reperfusion damage by dexmedetomidine in rat ovarian tissue. Gynecol Endocrinol 29: 222-225, 2013.

50. Hayashida K and Eisenach JC: Spinal $\alpha 2$-adrenoceptor-mediated analgesia in neuropathic pain reflects brain-derived nerve growth factor and changes in spinal cholinergic neuronal function. Anesthesiology 113: 406-412, 2010.

51. Peng L, Li B, Du T, et al: Astrocytic transactivation by alpha2A-adrenergic and 5-HT2B serotonergic signaling. Neurochem Int 57: 421-431, 2010.

52. Holers VM and Kulik L: Complement receptor 2, natura antibodies and innate immunity: Inter-relationships in B cell selection and activation. Mol Immunol 44: 64-72, 2007.

53. Fritz JH, Ferrero RL, Philpott DJ and Girardin SE: Nod-like proteins in immunity, inflammation and disease. Nat Immunol 7: 1250-1257, 2006.

54. Sezer A, Memis D, Usta U and Süt N: The effect of dexmedetomidine on liver histopathology in a rat sepsis model: an experimental pilot study. Ulus Travma Acil Cerrahi Derg 16 : 108-112, 2010.

55. Venn RM, Bryant A, Hall GM and Grounds RM: Effects of dexmedetomidine on adrenocortical function, and the cardiovascular, endocrine and inflammatory responses in post-operative patients needing sedation in the intensive care unit. Br J Anaesth 86: 650-656, 2001.

56. Tasdogan M, Memis D, Sut N and Yuksel M: Results of a pilot study on the effects of propofol and dexmedetomidine on inflammatory responses and intraabdominal pressure in severe sepsis. J Clin Anesth 21: 394-400, 2009.

57. Lin Y, Zhu X, Yao WZ, Yang YL, A LT and Chen L: Yohimbine protects against endotoxin-induced acute lung injury by blockade of alpha $2 \mathrm{~A}$ adrenergic receptor in rats. Chin Med J (Engl) 124 1069-1074, 2011

58. Kishikawa H, Kobayashi K, Takemori K, Okabe T, Ito K and Sakamoto A: The effects of dexmedetomidine on human neutrophil apoptosis. Biomed Res 29: 189-194, 2008.

59. Uysal HY, Cuzdan SS, Kayiran O, et al: Preventive effect of dexmedetomidine in ischemia-reperfusion injury. J Craniofac Surg 23: 1287-1291, 2012.

60. Lai YC, Tsai PS and Huang CJ: Effects of dexmedetomidine on regulating endotoxin-induced up-regulation of inflammatory molecules in murine macrophages. J Surg Res 154: 212-219, 2009.
61. Zhang H, Zhou F, Li C, et al: Molecular mechanisms underlying the analgesic property of intrathecal dexmedetomidine and its neurotoxicity evaluation: an in vivo and in vitro experimental study. PLoS One 8: e55556, 2013.

62. Peng M, Wang YL, Wang CY and Chen C: Dexmedetomidine attenuates lipopolysaccharide-induced proinflammatory response in primary microglia. J Surg Res 179: e219-e225, 2013.

63. Memiş D, Hekimoğlu S, Vatan I, Yandim T, Yüksel M and Süt N: Effects of midazolam and dexmedetomidine on inflammatory responses and gastric intramucosal $\mathrm{pH}$ to sepsis, in critically ill patients. Br J Anaesth 98: 550-552, 2007.

64. Polat B, Albayrak Y, Suleyman B, et al: Antiulcerative effect of dexmedetomidine on indomethacin-induced gastric ulcer in rats. Pharmacol Rep 63: 518-526, 2011.

65. Walker SM, Howard RF, Keay KA and Fitzgerald M: Developmental age influences the effect of epidural dexmedetomidine on inflammatory hyperalgesia in rat pups. Anesthesiology 102: 1226-1234, 2005.

66. Happe HK, Bylund DB and Murrin LC: Alpha(2)-adrenoceptor-stimulated GTP gamma S binding in rat brain: an autoradiographic study. Eur J Pharmacol 399: 17-27, 2000.

67. Jansson CC, Pohjanoksa K, Lang J, Wurster S, Savola JM and Scheinin M: Alpha2-adrenoceptor agonists stimulate high-affinity GTPase activity in a receptor subtype-selective manner. Eur J Pharmacol 374: 137-146, 1999.

68. Lawrence CJ, Prinzen FW and de Lange S: The effect of dexmedetomidine on nutrient organ blood flow. Anesth Analg 83: 1160-1165, 1996.

69. Geze S, Cekic B, Imamoğlu M, et al: Use of dexmedetomidine to prevent pulmonary injury after pneumoperitoneum in ventilated rats. Surg Laparosc Endosc Percutan Tech 22: 447-453, 2012.

70. Laudenbach V, Mantz J, Lagercrantz H, Desmonts JM, Evrard P and Gressens P: Effects of alpha(2)-adrenoceptor agonists on perinatal excitotoxic brain injury: comparison of clonidine and dexmedetomidine. Anesthesiology 96: 134-141, 2002.

71. Davies MF, Tsui J, Flannery JA, Li X, DeLorey TM and Hoffman BB: Activation of alpha2 adrenergic receptors suppresses fear conditioning: expression of c-Fos and phosphorylated CREB in mouse amygdala. Neuropsychopharmacology 29: 229-239, 2004.

72. Mimuro S, Katoh T, Suzuki A, et al: Deterioration of myocardial injury due to dexmedetomidine administration after myocardial ischaemia. Resuscitation 81: 1714-1717, 2010.

73. Willigers HM, Prinzen FW, Roekaerts PM, de Lange S and Durieux ME: Dexmedetomidine decreases perioperative myocardial lactate release in dogs. Anesth Analg 96: 657-664, 2003.

74. Okada H, Kurita T, Mochizuki T, Morita K and Sato S: The cardioprotective effect of dexmedetomidine on global ischaemia in isolated rat hearts. Resuscitation 74: 538-545, 2007.

75. Roekaerts PM, Prinzen FW and De Lange S: Beneficial effects of dexmedetomidine on ischaemic myocardium of anaesthetized dogs. Br J Anaesth 77: 427-429, 1996.

76. Kocoglu H, Ozturk H, Ozturk H, Yilmaz F and Gulcu N: Effect of dexmedetomidine on ischemia-reperfusion injury in rat kidney: a histopathologic study. Ren Fail 31: 70-74, 2009.

77. Marangoni MA, Hausch A, Vianna PT, Braz JR, Viero RM and Castiglia YM: Renal function and histology after acute hemorrhage in rats under dexmedetomidine action. Acta Cir Bras 22: 291-298, 2007.

78. Villela NR, do Nascimento Júnior P, de Carvalho LR and Teixeira A: Effects of dexmedetomidine on renal system and on vasopressin plasma levels. Experimental study in dogs. Rev Bras Anestesiol 55: 429-440, 2005.

79. Frumento RJ, Logginidou HG, Wahlander S, Wagener G, Playford HR and Sladen RN: Dexmedetomidine infusion is associated with enhanced renal function after thoracic surgery. J Clin Anesth 18: 422-426, 2006.

80. Bayram A, Esmaoglu A, Akin A, et al: The effects of intraoperative infusion of dexmedetomidine on early renal function after percutaneous nephrolithotomy. Acta Anaesthesiol Scand 55: 539-544, 2011.

81. Yang CL, Chen CH, Tsai PS, Wang TY and Huang CJ: Protective effects of dexmedetomidine-ketamine combination against ventilator-induced lung injury in endotoxemia rats. J Surg Res 167: e273-e281, 2011.

82. Hanci V, Erol B, Bektas S, et al: Effect of dexmedetomidine on testicular torsion/detorsion damage in rats. Urol Int 84: 105-111, 2010. 
83. Gu J, Chen J, Xia P, Tao G, Zhao H and Ma D: Dexmedetomidine attenuates remote lung injury induced by renal ischemia-reperfusion in mice. Acta Anaesthesiol Scand 55: 1272-1278, 2011.

84. Sanders RD, Xu J, Shu Y, et al: Dexmedetomidine attenuates isoflurane-induced neurocognitive impairment in neonatal rats Anesthesiology 110: 1077-1085, 2009.

85. Bekker A, Haile M, Kline R, et al: The effect of intraoperative infusion of dexmedetomidine on the quality of recovery after major spinal surgery. J Neurosurg Anesthesiol 25: 16-24, 2013.

86. Kuhmonen J, Pokorný J, Miettinen R, et al: Neuroprotective effects of dexmedetomidine in the gerbil hippocampus after transient global ischemia. Anesthesiology 87: 371-377, 1997.

87. Goyagi T, Nishikawa T, Tobe Y and Masaki Y: The combined neuroprotective effects of lidocaine and dexmedetomidine after transient forebrain ischemia in rats. Acta Anaesthesiol Scand 53 1176-1183, 2009.

88. Sato K, Kimura T, Nishikawa T, Tobe $\mathrm{Y}$ and Masaki Y: Neuroprotective effects of a combination of dexmedetomidine and hypothermia after incomplete cerebral ischemia in rats. Acta Anaesthesiol Scand 54: 377-382, 2010.
89. Ma D, Hossain M, Rajakumaraswamy N, et al: Dexmedetomidine produces its neuroprotective effect via the alpha $2 \mathrm{~A}$-adrenoceptor subtype. Eur J Pharmacol 502: 87-97, 2004.

90. Benggon M, Chen H, Applegate R, Martin R and Zhang JH Effect of dexmedetomidine on brain edema and neurological outcomes in surgical brain injury in rats. Anesth Analg 115: 154-159, 2012.

91. Gul S, Hanci V, Bahadir B, et al: The effectiveness of dexmedetomidine in experimental spinal cord injury compared to methylprednisolone in rats. J Clin Neurosci 17: 490-494, 2010.

92. Can M, Gul S, Bektas S, Hanci V and Acikgoz S: Effects of dexmedetomidine or methylprednisolone on inflammatory responses in spinal cord injury. Acta Anaesthesiol Scand 53: 1068-1072, 2009.

93. Aslan A, Cemek M, Eser O, et al: Does dexmedetomidine reduce secondary damage after spinal cord injury? An experimental study. Eur Spine J 18: 336-344, 2009.

94. Bell MT, Puskas F, Smith PD, et al: Attenuation of spinal cord ischemia-reperfusion injury by specific $\alpha$-2a receptor activation with dexmedetomidine. J Vasc Surg 56: 1398-1402, 2012. 